\title{
Proprioceptive neuromuscular facilitation stretching combined with Kinesio taping for hamstring flexibility in amateur athletes: a single-blind, randomized, controlled trial
}

\author{
DOI: https://doi.org/10.5114/pq.2020.100295
}

\author{
Dilber Karagozoglu Coskunsu', Ebru Kaya Mutlu², Arzu Razak Ozdincler ${ }^{3}$ \\ ${ }^{1}$ Department of Physiotherapy and Rehabilitation, Faculty of Health Sciences, Fenerbahçe University, Istanbul, Turkey \\ 2 Department of Physiotherapy and Rehabilitation, Faculty of Health Sciences, Istanbul University-Cerrahpaşa, Istanbul, Turkey \\ ${ }^{3}$ Department of Physiotherapy and Rehabilitation, Faculty of Health Sciences, Biruni University, Istanbul, Turkey
}

\section{Abstract}

Introduction. This study investigated the effect of proprioceptive neuromuscular facilitation (PNF), Kinesio® tape (KT), and PNF combined with KT (PNF + KT) for hamstring flexibility and jumping performance in amateur athletes with hamstring tightness.

Methods. Overall, 128 amateur athletes were randomly assigned to the PNF $(n=32), \mathrm{KT}(n=32), \mathrm{PNF}+\mathrm{KT}(n=32)$ or control group $(n=32)$. Flexibility was assessed with active straight leg raise (ASLR) test with a digital goniometer. Performance was evaluated by vertical jump (VJ) and triple hop distance (THD) tests. All measurements were performed by a blinded assessor 3 times: at baseline, immediately after the interventions, and 30 minutes after the interventions.

Results. Amateur athletes receiving PNF or KT or PNF + KT demonstrated a greater increase in ASLR and THD tests from baseline to post-intervention scores $(p=0.001, p=0.002, p=0.001$ for ASLR; $p=0.001$ in all groups for THD, respectively) and from baseline to 30 minutes after intervention ( $p=0.001$ in all groups for ASLR; $p=0.001$ in all groups for THD, respectively). Nevertheless, only the PNF + KT group presented a greater increase in VJ test from baseline to post-intervention scores $(p=0.02)$. Besides, the effect sizes for the PNF + KT group were higher than in the other groups in all parameters.

Conclusions. PNF + KT might be more effective in increasing hamstring flexibility and jumping performance in amateur athletes with hamstring tightness than each method alone.

Key words: flexibility, proprioceptive neuromuscular facilitation, Kinesio tape, athletes, range of motion

\section{Introduction}

Flexibility is vital for all movements, and changes in flexibility may cause abnormal loading of the musculoskeletal system [1] and injuries [1, 2]. Insufficient hamstring flexibility has been shown to be related to problems such as muscle imbalances, muscle injuries, patellar tendinopathy and patellofemoral pain, and low back pain [3]. Many methods are used to reduce the tightness of the hamstring muscles and increase athletic performance and range of motion (ROM); these include static and ballistic stretching, proprioceptive neuromuscular facilitation (PNF) [4, 5], Kinesio ${ }^{\circledR}$ tape (KT) applications [6], muscle energy techniques [7], foam roller [1], and instrument-assisted soft tissue mobilizations [8] in various interventions and training programs. However, there is still insufficient evidence about which technique is more effective.

PNF stretching techniques are commonly used in athletic and clinical environments to enhance ROM with a view to optimizing motor performance and rehabilitation. PNF stretching incorporates static stretching and isometric contractions in a cyclical pattern to enhance ROM with 3 techniques commonly referred to in the literature, namely contract relax, hold relax, and contract relax agonist contract [9]. Although the mechanism of PNF remains unclear, its ability to increase both active and passive ROM has been proved $[10,11]$. However, the effect of PNF on performance is still controversial. Although a systematic review suggested that increasing eccentric and concentric peak torque enhanced hamstring performance [12] after PNF, there are also some studies reporting a reduction in the maximal voluntary isometric contraction force [13], isokinetic peak torque of hamstrings, and vertical jump performance [14].

On the other hand, the KT technique, which was developed by Dr. Kenzo Kase, has been commonly used in rehabilitation of musculoskeletal injuries and improved athletic performance. KT differs from other taping methods with its flexibility and structural features similar to human skin, and allows joint movement [15]. KT has been applied in sports rehabilitation for correcting or increasing muscle activity, improving active ROM [16], decreasing pain, repositioning joints [17].

As mentioned above, there is information about the effect of PNF and KT applications on ROM and performance. However, there is no study comparing the advantages of PNF and KT in which these two are used together to investigate the immediate effect on hamstring flexibility and jumping performance in amateur athletes with hamstring tightness. In this study, we aimed to increase flexibility and performance with the combined use of the contract relax PNF technique, which employs autogenic inhibition techniques, and the fascial KT technique, which is thought to provide fascia correction. With

Correspondence address: Ebru Kaya Mutlu, Department of Physiotherapy and Rehabilitation, Faculty of Health Sciences, Istanbul University-Cerrahpaşa, Büyükçekmece Yerleşkesi Alkent 2000 Mahallesi Yiğittürk Caddesi No: 5/9/1, 34500 Büyükçekmece/Istanbul, Turkey, e-mail: fztebrukaya@hotmail.com

Citation: Coskunsu DK, Mutlu EK, Ozdincler AR. Proprioceptive neuromuscular facilitation stretching combined with Kinesio taping for hamstring flexibility in amateur athletes: a single-blind, randomized, controlled trial. Physiother Quart. 2021;29(3):56-61; doi: https://doi. org/10.5114/pq.2020.100295. 
these techniques, the muscles were stretched and loosed and then afferent stimulation was applied on the fascia. Implementing a technique that improves performance together with stretching techniques which increase flexibility can enhance muscle performance and reduce the risk of injury to hamstring muscles. We therefore hypothesized that PNF combined with KT would be more effective for improving ROM and jumping performance. The purpose of the study was to investigate the effect of PNF, KT, and PNF combined with $\mathrm{KT}(\mathrm{PNF}+\mathrm{KT})$ on ROM and jumping performance.

\section{Subjects and method}

\section{Study design}

The research was designed as a randomized, multicentre, single-blind, case-control study. It was performed at Bahçeşehir University, Istanbul University-Cerrahpaşa, and Biruni University between February and July 2019, after being announced by posting information to notice boards of the above-mentioned universities and with written notices sent to sports clubs of the universities.

The participants who met the study criteria were randomly assigned to 1 of the 4 intervention groups (ratio: 1:1:1:1): $\mathrm{PNF}, \mathrm{KT}, \mathrm{PNF}+\mathrm{KT}$, and control. Randomization was carried out with a random number list prepared by an investigator with no clinical involvement in the study by using a randomization website (https://www.randomizer.org/). Numbered index cards (1-128) were folded and placed in sealed opaque envelopes. Next, another investigator (E.K.M.) opened each envelope and assigned the participants to the groups depending on the selected index card.

All evaluations were conducted by an investigator (D.K.C.) who had a clinical experience of more than 20 years in orthopaedics and sports rehabilitation and who was blinded by staying outside the laboratory while the athletes were undergoing the interventions. Also, to prevent the assessor from understanding the applied intervention, the athletes were dressed in dark long tights so that KT was not seen.

\section{Subjects}

A total of 132 amateur athletes volunteered to participate in this study; 128 of them met the inclusion criteria: they were aged 18-30 years, as well as had hamstring tightness confirmed by the fingertip-to-floor test (if the third finger was unable to touch the ground) [17] and by the passive knee extension test (if the angle was $\geq 19.2^{\circ}$ in women, $\geq 32.2^{\circ}$ in men) [2]. Subjects were excluded in the case of pregnancy, surgery in the area of lower extremity or pelvis within the previous 6 months, history of a lower extremity trauma, history of hamstring strain or triceps surae strain, allergic reactions to $\mathrm{KT}$, or skin disease.

All interventions and assessments were performed on the dominant sides of the athletes. Dominance was determined with kicking a ball.

\section{Evaluations}

The primary outcome of this study was the active straight leg raise (ASLR) test. Secondary outcomes were the vertical jump (VJ) test and the triple hop distance (THD) test.

All evaluations were performed at baseline, immediately after, and 30 minutes after the interventions in the PNF, KT, and PNF + KT groups, whereas at baseline and 30 minutes after the baseline evaluation in the control group. After jog- ging for 5 minutes to warm up, evaluation was started with the $\mathrm{VJ}$ and THD tests to investigate the effect of hamstring tightness on the performance. Then, hamstring tightness of the dominant side was measured with the ASLR test.

\section{Vertical jump test}

The countermovement VJ test was applied as described by Bradley et al. [18]. The VJ height was measured by calculating the distance between the marks while standing flat footed next to a wall with the arm extending upward and the maximum height reached by jumping. All volunteers jumped 3 times with a minimum interval of 45 seconds between the jumps, and the biggest height value was taken into consideration. The test was repeated if the marked point was lost or the athlete jumped in a wrong position.

\section{Triple hop distance test}

THD was performed as described by Bolgla and Keskula [19]. A test trial was repeated if the participant was unable to complete a triple hop without losing balance and contacted the ground with the opposite leg. The mean value of the maximum distances recorded after 3 test trials in centimetres was used for analysis [20].

\section{Active straight leg raise test}

The test was performed in supine position as described by Mason et al. [21]. The flexion range of the hip joint was measured with a digital goniometer.

\section{Interventions}

\section{Proprioceptive neuromuscular facilitation}

The contract relax PNF stretching technique was started when the patients were in supine position with both legs in extension. The investigator took the dominant leg slowly to the end of the passive ROM. Then, the athletes were asked to contract the hamstring for a $20 \%$ of maximal voluntary contraction for a period of 10 seconds against the resistance of the investigator's shoulder [8]. Immediately after the 10-second contraction, the investigator maintained the position, instructing the athlete to relax. Then, the athlete moved their leg actively to the new limit of ROM. At the new end point, passive stretch was held for 30 seconds [8]. This procedure was repeated 4 times [8]. All PNF interventions were performed by a certified investigator (A.R.O.).

\section{Kinesio ${ }^{\circledR}$ tape intervention}

KT with a width of $5 \mathrm{~cm}$ was applied to the hamstring muscles with the fascia technique from ischial tuberosity to the back of the knee with $25 \%$ tension while the volunteers were in standing position with their trunk bent, keeping their knee extended. Then, the Y-shaped tape was applied around the lateral and medial sides of the knee [22] (Figure 1). All KT interventions were performed by an investigator (E.K.M.) holding an internationally recognized certificate.

\section{Proprioceptive neuromuscular facilitation \\ + Kinesio $^{\circledR}$ tape intervention}

First PNF and then KT, immediately after PNF, were applied to the hamstring muscles as explained above. 


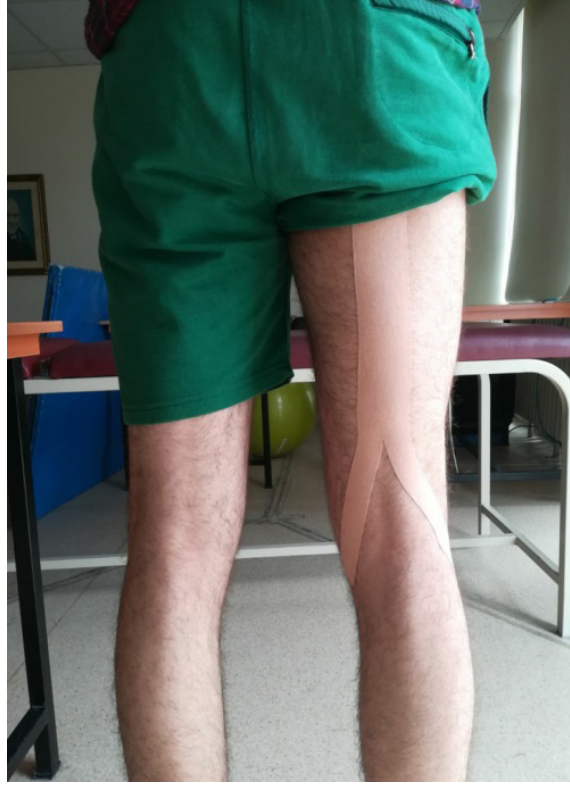

Figure 1. Kinesio ${ }^{\circledR}$ tape application

Control group

No intervention was implemented in the control group. All evaluations were performed at baseline and 30 minutes after the baseline assessment.

\section{Sample size}

The sample size was calculated by using the Raosoft Sample Size Calculator program. On the basis of the number of amateur athletes $(4,907,955)$ (https://shgm.gsb.gov. tr/Sayfalar/175/105/Istatistikler) and the population of Turkey (80 million), the percentage of amateur athletes was found to be $8 \%$. With the assumption of a $95 \%$ confidence interval and $95 \%$ power, the resulting sample size was a total of 112 individuals, with 28 participants per group. The estimated drop-out rate was $20 \%$; the number of patients required for recruitment was calculated as 128 .

\section{Statistical analysis}

The study data were evaluated with the Statistical Package for the Social Sciences 20.0 program. Before the statisti- cal analysis, the Kolmogorov-Smirnov test was used to assess the distribution of data. Our data were found to be normally distributed, so a parametric test was applied for statistical analysis. Demographic comparisons of the 4 groups were conducted with the chi-square analysis for categorical variables and the ANOVA for continuous variables. One-way analysis of covariance (ANCOVA) was used, with baseline values as a covariate, to test the effect of applications on the ASLR, VJ, and THD tests at each time interval (after the application and after 30 minutes) as the within-subject variable and group (PNF, KT, and PNF + KT) as the betweensubject variable using the Bonferroni equality at an alpha level of 0.05 . Effect sizes were determined as suggested by Kazis et al. [23], i.e. by dividing the changes in mean baseline and follow-up scores by the baseline standard deviation. The effect sizes of $0.2,0.5$, and 0.8 were considered small, moderate, and large, respectively.

\section{Ethical approval}

The research related to human use has complied with all the relevant national regulations and institutional policies, has followed the tenets of the Declaration of Helsinki, and has been approved by the Research Ethics Committee of the Bahçeşehir University (IRB: 22481095-020-328).

\section{Informed consent}

Informed consent has been obtained from all individuals included in this study.

\section{Results}

One participant in the KT group, 2 in the PNF + KT group, and 4 control subjects discontinued the intervention; therefore, 121 subjects were analysed. The demographic data of the participants are presented in Table 1.

Participants receiving PNF, KT, or PNF + KT demonstrated a greater increase in ASLR and THD scores from baseline to post-intervention values and from baseline to 30 minutes after the intervention. Nevertheless, only the PNF + KT group presented a greater increase in the $\mathrm{VJ}$ test from baseline to post-intervention scores (Table 2).

A one-way between-group analysis of covariance was conducted to compare the effectiveness of the 4 different interventions. Preliminary checks were performed to ensure that there was no violation of the assumptions of normality, linearity, homogeneity of variances, homogeneity of regres-

Table 1. Socio-demographic characteristics

\begin{tabular}{|c|c|c|c|c|c|}
\hline Characteristics & $\begin{array}{l}\text { PNF group } \\
(n=32)\end{array}$ & $\begin{array}{l}\text { KT group } \\
(n=31)\end{array}$ & $\begin{array}{c}\text { PNF + KT group } \\
\quad(n=30)\end{array}$ & $\begin{array}{l}\text { Control group } \\
\qquad(n=28)\end{array}$ & $p$ \\
\hline Age (years) [mean $(S D)]$ & $21.15(2.18)$ & $20.93(1.56)$ & $20.83(2.00)$ & $20.75(2.20)$ & $0.87^{*}$ \\
\hline Sex ( $n$, male/female) & $30 / 2$ & $26 / 5$ & $26 / 4$ & $20 / 8$ & $0.12^{\#}$ \\
\hline BMI $\left(\mathrm{kg} / \mathrm{m}^{2}\right)$ [mean $\left.(S D)\right]$ & $22.16(1.98)$ & $24.45(3.71)$ & $21.73(2.25)$ & $22.85(3.16)$ & $0.02^{*}$ \\
\hline $\begin{array}{l}\text { Sports branch [n (\%)] } \\
\text { Football } \\
\text { Basketball } \\
\text { Volleyball } \\
\text { Other }\end{array}$ & $\begin{array}{c}15(46.9) \\
4(12.5) \\
8(25) \\
5(15.6)\end{array}$ & $\begin{array}{c}14(45.2) \\
2(6.5) \\
11(35.5) \\
4(12.9)\end{array}$ & $\begin{array}{c}20(66.7) \\
4(13.3) \\
5(16.7) \\
1(3.3)\end{array}$ & $\begin{array}{c}19(67.9) \\
5(17.9) \\
3(10.7) \\
1(3.6)\end{array}$ & $0.17^{\#}$ \\
\hline $\begin{array}{l}\text { Dominant side }[n(\%)] \\
\text { Right } \\
\text { Left }\end{array}$ & $\begin{array}{c}25(78.1) \\
7(21.9)\end{array}$ & $\begin{array}{l}17(54.8) \\
14(45.2)\end{array}$ & $\begin{array}{c}21(70) \\
9(30)\end{array}$ & $\begin{array}{c}25(89.3) \\
3(10.7)\end{array}$ & $0.02^{\#}$ \\
\hline
\end{tabular}

PNF - proprioceptive neuromuscular facilitation, KT - Kinesio ${ }^{\circledR}$ taping, BMI - body mass index

* one-way ANOVA, \# chi-square test

Values of $p<0.05$ are marked with bold. 
Table 2. A comparison of active straight leg raise test, vertical jump test, and triple hop distance test between the groups at baseline, after the application, and after 30 minutes

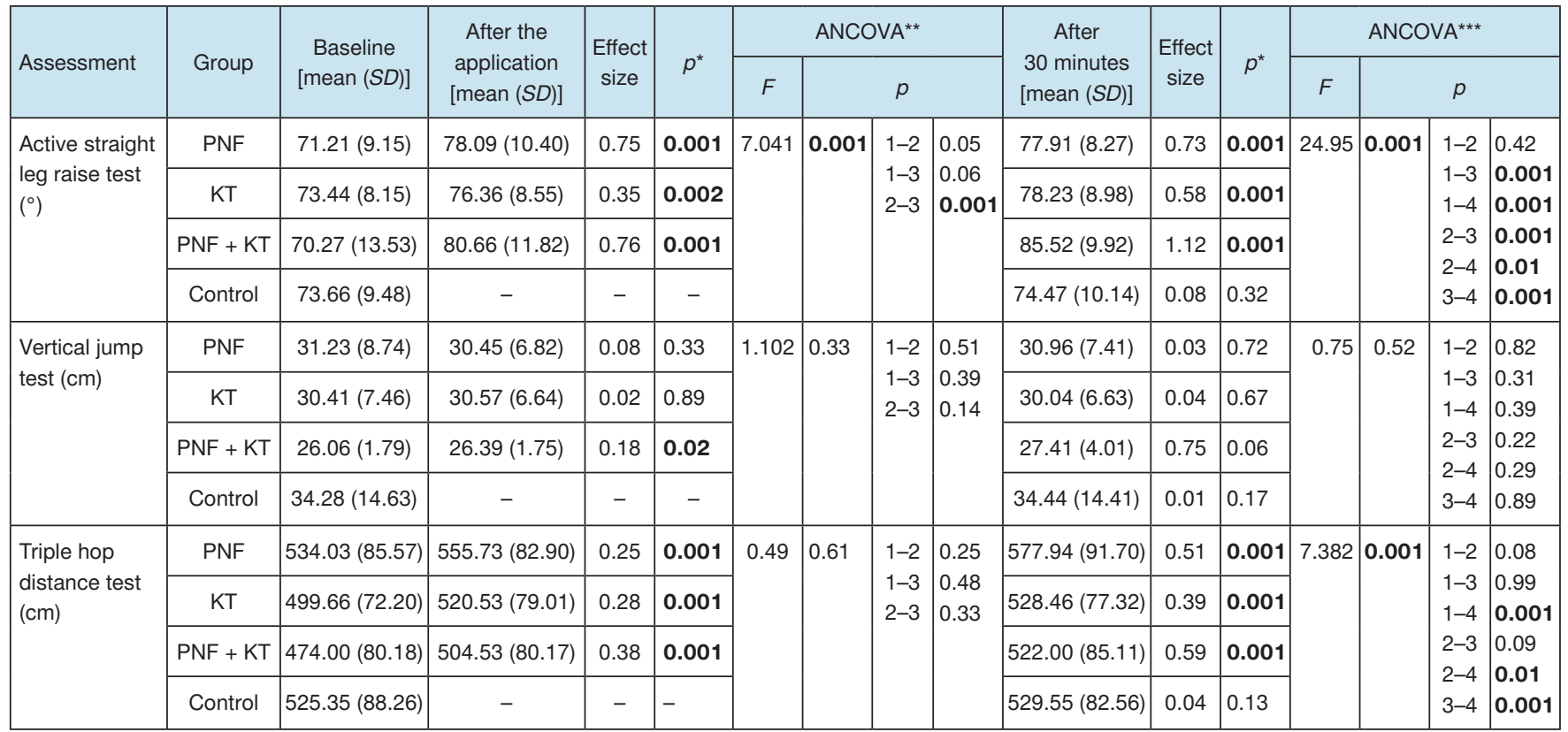

PNF - proprioceptive neuromuscular facilitation, KT - Kinesio ${ }^{\circledR}$ taping

* paired sample $t$-test

${ }^{* *}$ statistically significant between-group differences after the application, with baseline value as a covariate (ANCOVA, $p<0.05$ )

*** statistically significant between-group differences after 30 minutes, with baseline value as a covariate (ANCOVA, $p<0.05)$

Values of $p<0.05$ are marked with bold.

sion slopes, and reliable measurement of the covariate. After adjusting for baseline, there was a significant difference between the 4 intervention groups in ASLR and THD after 30 minutes $(F=24.951, p=0.001$, partial eta squared: 0.39 ; $F=7.382, p=0.001$, partial eta squared: 0.16 , respectively). A strong relationship was observed between baseline and scores after 30 minutes in ASLR and THD, as indicated by the partial eta squared value of 0.58 and 0.80 , respectively (Table 2). On the other hand, there was no statistically significant between-group difference in the VJ test (Table 2).

\section{Discussion}

This single-blind randomized controlled trial reveals that combining PNF stretching with KT results in slightly better immediate effects as compared with PNF alone or KT alone in terms of ASLR in amateur athletes. Additionally, the effect size was large for PNF + KT (effect size: 1.12) in ASLR. Only the PNF + KT procedure was effective in the VJ height 30 minutes after application. Regarding THD, there was a statistically significant increase in all intervention groups but not in the control group immediately after and 30 minutes after the intervention.

Results of several studies [4, 5, 24] support the use of PNF stretching to increase hamstring flexibility. A systemic review [12] and a meta-analysis [11] which compare different stretching techniques (static, ballistic, PNF) report that all techniques are effective in improving hamstring flexibility and none has advantage over another. There is a review [9] arguing that PNF stretching is the most effective method for increasing ROM, especially in short-term recoveries. It was shown in that review that even 1 session of PNF stretching increased ROM by $3-9^{\circ}$, depending on the joint, and this effect could continue up to 90 minutes. The mean $6.88^{\circ}$ recovery in ROM obtained in the PNF stretching group in our study is compatible with the literature data. We explain the increase in ROM in PNF applications with the autogenic in- hibition mechanism [25]. The mechanism of action of the contract relax technique used in our study is that isometric contractions applied to the stretched (antagonist) muscle lead to increased tension on the Golgi tendon organ, which triggers an autogenic inhibition mechanism overcoming the myotatic reflex and reducing the neural activity in the stretched muscle [26].

The impact of PNF stretching techniques on performance is controversial. The literature concludes that PNF decreases the performance in maximum effort exercises when completed prior to exercise, but increases athletic performance when it is performed consistently and after exercise [25]. Bradley et al. [18] stated that after applying contract relax stretching to hamstrings, there was a $5 \%$ decrease in VJ performance and the results returned to normal values $15 \mathrm{~min}$ utes later. However, in our study, although it was not statistically significant, we observed a decrease in VJ height after the intervention and the VJ height did not return to the baseline values in the assessment performed 30 minutes after the intervention. The duration and the number of repetitions of the PNF stretching technique used in our study may be a reason for these different results. While Bradley et al. [18] applied the contract relax technique with 4 repetitions, $5 \mathrm{sec}$ onds of maximal isometric contraction and 25 seconds of passive stretching, we performed a protocol involving 4 repetitions, 10 seconds of contraction in $20 \%$ of maximal voluntary isometric contraction, and 30 seconds of passive stretching. The fact that there was no increase in the VJ height in the PNF group is also consistent with the results of a review [27] arguing that acute stretching does not increase the $\mathrm{VJ}$ height.

The second test used for performance analysis in our study was THD. In the literature, there is no study evaluating the hop distance after PNF stretching application. On the other hand, while PNF stretching did not affect the VJ height, it increased the THD test scores; this may be due to the fact that these 2 performance tests are based on different parame- 
ters. VJ evaluates the explosive power parameter, and THD is affected by the parameters of power, balance, and speed [28].

Considering the mechanism of action of the KT technique, Kase et al. [15] suggested that KT increased the space between the skin and muscle tissue, lifting the skin; in this way, this technique reduces pain by increasing blood and lymphatic flow and increases ROM by normalizing muscle function. Nevertheless, studies investigating this issue have yielded contradictory results. Merino-Marban et al. [6] investigated the acute effect of KT on hamstring extensibility in healthy students without hamstring tightness and reported that the technique did not increase hip flexion ROM. On the other hand, another study examining the acute effect of KT applied to quadriceps and hamstring muscles of healthy subjects without tightness implied that KT increased hip and knee ROM [17]. Similarly to Flood et al. [16], we conducted our study among athletes with hamstring tightness. ASLR statistically significantly increased immediately after and 30 minutes after the KT intervention. In turn, we found that PNF $+K T$ was more effective than the other interventions.

Although hamstrings are among the prime movers in jump tests [19], we have not found any study examining the effect of KT on hamstring muscles in jumping performance. Harput et al. [29] found an increase in single-leg hop distance performance immediately after applying Y-shaped KT to the quadriceps muscle in people who had undergone anterior cruciate ligament reconstruction. Aktas and Baltaci [30] reported an improvement in single-leg hop distance, which is similar to our study, but there was no change in VJ height immediately after Y-shaped KT application to the quadriceps muscle in healthy individuals. Another study suggested that KT applied to the triceps surae muscles did not have a significant effect on VJ height [31]. VJ tests, regardless of performance with 1 leg or 2 legs, as in our study, contain less balance components than single hop or THD tests. Our results and the results found in the literature may be due to an increased balance resulting from the cutaneous stimulation created by KT application on the skin. However, we suppose that the larger effect sizes obtained in THD in the PNF + KT group compared with the other groups may be due to the improvement of ROM achieved with PNF stretching.

In the PNF + KT combination, KT relaxes the fascia, while the PNF stretching technique relaxes the muscle by autogenic inhibition of the muscle's Golgi tendon organ; the 2 effects combine to increase flexibility [9, 15]. Consistent with our hypothesis, the greatest effect in ASLR measurements, especially after 30 minutes, was observed in the PNF + KT group in our study. The fact that there was an increase in VJ height only in the evaluation performed 30 minutes later and only in the PNF + KT group suggests that the combination of these 2 methods may have a positive effect on jump performance. Similarly, the greatest effect in THD was also seen in the $\mathrm{PNF}+\mathrm{KT}$ group.

To our knowledge, this is the first study to assess the combination of PNF and KT in hamstring tightness. Most of the studies in literature which investigated the effect of PNF or KT were conducted among healthy subjects, but in the present study, the participants were amateur athletes with hamstring tightness. The fact that the interventions and evaluations were carried out by researchers experienced in their field was another strength of our study.

\section{Limitations}

This study has some limitations. First, the assessment of after the interventions, but no long-term result was observed. Second, owing to numerical deficiencies, we could not conduct the performance evaluations specific to the athletes' sports branches.

\section{Conclusions}

This single-blind randomized controlled trial demonstrated that PNF, KT, and PNF + KT had a similar effect on the flexibility of hamstring and performance. Beyond statistical significance, the clinical interpretation of these findings implies that PNF + KT might provide a slightly better improvement in the flexibility of hamstring and performance than the PNF and KT interventions alone. Further studies are needed to support our findings.

\section{Disclosure statement}

No author has any financial interest or received any financial benefit from this research.

\section{Conflict of interest}

The authors state no conflict of interest.

\section{References}

1. Grieve R, Goodwin F, Alfaki M, Bourton A-J, Jeffries C, Scott $H$. The immediate effect of bilateral self myofascial release on the plantar surface of the feet on hamstring and lumbar spine flexibility: a pilot randomised controlled trial. J Bodyw Mov Ther. 2015;19(3):544-552; doi: 10.1016/ j.jbmt.2014.12.004.

2. Yıldırım MŞ, Tuna F, Kabayel DD, Süt N. The cut-off values for the diagnosis of hamstring shortness and related factors. Balkan Med J. 2018;35(5):388-393; doi: 10.4274/balkanmedj.2017.1517.

3. Medeiros DM, Cini A, Sbruzzi G, Lima CS. Influence of static stretching on hamstring flexibility in healthy young adults: systematic review and meta-analysis. Physiother Theory Pract. 2016;32(6):438-445; doi: 10.1080/09593 985.2016.1204401.

4. Yıldırım MS, Ozyurek S, Tosun OÇ, Uzer S, Gelecek N. Comparison of effects of static, proprioceptive neuromuscular facilitation and Mulligan stretching on hip flexion range of motion: a randomized controlled trial. Biol Sport.2016;33(1):89-94;doi:10.5604/20831862.1194126.

5. Khodayari B, Dehghani Y. The investigation of mid-term effect of different intensity of PNF stretching on improve hamstring flexibility. Procedia Soc Behav Sci. 2012;46: 5741-5744; doi: 10.1016/j.sbspro.2012.06.508.

6. Merino-Marban R, Fernandez-Rodriguez E, Lopez-Fernandez I, Mayorga-Vega D. The acute effect of Kinesio taping on hamstring extensibility in university students. J Phys Educ Sport. 2011;11(2):133-137.

7. Smith M, Fryer G. A comparison of two muscle energy techniques for increasing flexibility of the hamstring muscle group. J Bodyw Mov Ther. 2008;12(4):312-317; doi: 10.1016/j.jbmt.2008.06.011.

8. Gunn LJ, Stewart JC, Morgan B, Metts ST, Magnuson JM, Iglowski NJ, et al. Instrument-assisted soft tissue mobilization and proprioceptive neuromuscular facilitation techniques improve hamstring flexibility better than static stretching alone: a randomized clinical trial. J Man ManipTher.2019;27(1):15-23;doi:10.1080/10669817.2018. 1475693.

9. Sharman MJ, Cresswell AG, Riek S. Proprioceptive neuromuscular facilitation stretching: mechanisms and clinical implications. Sports Med. 2006;36(11):929-939; doi: 10.2165/00007256-200636110-00002. 
10. Cayco CS, Labro AV, Gorgon EJR. Hold-relax and contract-relax stretching for hamstrings flexibility: a systematic review with meta-analysis. Phys Ther Sport. 2019; 35:42-55; doi: 10.1016/j.ptsp.2018.11.001.

11. Decoster LC, Cleland J, Altieri C, Russell P. The effects of hamstring stretching on range of motion: a systematic literature review. J Orthop Sports Phys Ther. 2005;35(6): 377-387; doi: 10.2519/jospt.2005.35.6.377.

12. Rubini EC, Costa ALL, Gomes PSC. The effects of stretching on strength performance. Sports Med. 2007;37(3): 213-224; doi: 10.2165/00007256-200737030-00003.

13. Brandenburg JP. Duration of stretch does not influence the degree of force loss following static stretching. $J$ Sports Med Phys Fitness. 2006;46(4):526-534.

14. Cervantes SJ, Snyder AR. The effectiveness of a dynamic warm-up in improving performance in college athletes. J Sport Rehabil. 2011;20(4):487-493; doi: 10.1123/ jsr.20.4.487.

15. Kase K, Wallis J, Kase T. Clinical therapeutic applications of the Kinesio taping method. Albuquerque: Kinesio Taping Association; 2003.

16. Flood LT, Hassler RL, Sykora JL. The effects of Kinesio tape on range of motion, power output, and strength in female collegiate club athletes. Int J Res Ex Phys. 2016; 11(2):51-67.

17. Lemos TV, Albino ACG, Matheus JPC, de Melo Barbosa $A$. The effect of Kinesio taping in forward bending of the lumbar spine. J Phys Ther Sci. 2014;26(9):13711375; doi: 10.1589/jpts.26.1371.

18. Bradley PS, Olsen PD, Portas MD. The effect of static, ballistic, and proprioceptive neuromuscular facilitation stretching on vertical jump performance. J Strength Cond Res. 2007;21(1):223-226; doi: 10.1519/00124278-200 702000-00040.

19. Bolgla LA, Keskula DR. Reliability of lower extremity functional performance tests. J Orthop Sports Phys Ther. 1997;26(3):138-142; doi: 10.2519/jospt.1997.26.3.138.

20. Hamilton RT, Shultz SJ, Schmitz RJ, Perrin DH. Triplehop distance as a valid predictor of lower limb strength and power. J Athl Train. 2008;43(2):144-151; doi: 10.4085/ 1062-6050-43.2.144.

21. Mason JS, Crowell M, Dolbeer J, Morris J, Terry A, Koppenhaver $S$, et al. The effectiveness of dry needling and stretching vs. stretching alone on hamstring flexibility in patients with knee pain: a randomized controlled trial. Int J Sports Phys Ther. 2016:11(5):672-683.

22. Mutlu EK, Mustafaoglu R, Birinci T, Ozdincler AR. Does Kinesio taping of the knee improve pain and functionality in patients with knee osteoarthritis? A randomized controlled clinical trial. Am J Phys Med Rehabil. 2017;96(1): 25-33; doi: 10.1097/PHM.0000000000000520.

23. Kazis LE, Anderson JJ, Meenan RF. Effect sizes for interpreting changes in health status. Med Care. 1989; 27(3 Suppl.):178-189; doi: 10.1097/00005650-1989030 01-00015.

24. Kumar GP. Comparison of cyclic loading and hold relax technique in increasing resting length of hamstring muscles. Hong Kong Physiother J. 2011;29(1):31-33; doi: 10.1016/j.hkpj.2011.03.002.

25. Hindle KB, Whitcomb TJ, Briggs WO, Hong J. Proprioceptive neuromuscular facilitation (PNF): its mechanisms and effects on range of motion and muscular function. J Hum Kinet. 2012;31:105-113; doi: 10.2478/v100 78-012-0011-y.

26. Rowlands AV, Marginson VF, Lee J. Chronic flexibility gains: effect of isometric contraction duration during pro- prioceptive neuromuscular facilitation stretching techniques. Res Q Exerc Sport. 2003;74(1):47-51; doi: 10.1080/02701367.2003.10609063.

27. Shrier I. Does stretching improve performance? A systematic and critical review of the literature. Clin J Sport Med. 2004;14(5):267-273; doi: 10.1097/00042752$200409000-00004$.

28. Reiman MP, Manske RC. Functional testing in human performance. Champaign: Human Kinetics; 2009.

29. Harput G, Ulusoy B, Ozer H, Baltaci G, Richards J. External supports improve knee performance in anterior cruciate ligament reconstructed individuals with higher kinesiophobia levels. Knee. 2016;23(5):807-812; doi: 10.1016/j.knee.2016.05.008.

30. Aktas G, Baltaci G. Does Kinesiotaping increase knee muscles strength and functional performance? Isokinet Exerc Sci. 2011;19(3):149-155; doi: 10.3233/IES-20110408.

31. Huang C-Y, Hsieh T-H, Lu S-C, Su F-C. Effect of the Kinesio tape to muscle activity and vertical jump performance in healthy inactive people. BioMed Eng OnLine. 2011;10(1):70; doi: 10.1186/1475-925X-10-70. 